\title{
Voltage-driven superconducting weak link as a refrigerator for cooling of nanomechanical vibrations
}

\author{
G. Sonne, ${ }^{a)}$ M. E. Peña-Aza, and R. I. Shekhter \\ University of Gothenburg, Department of Physics, Göteborg SE-412 96, Sweden \\ L. Y. Gorelik \\ Chalmers University of Technology, Department of Applied Physics, Göteborg SE-412 96, Sweden
}

\section{Jonson}

University of Gothenburg, Department of Physics, Göteborg SE-412 96, Sweden, Heriot-Watt University, School of Engineering and Physical Sciences, Edinburgh EH14 4AS, Scotland, United Kingdom and Konkuk University, School of Physics, Division of Quantum Phases and Devices, Seoul 143-107, South Korea

(Submitted March 31, 2010)

Fiz. Nizk. Temp. 36, 1128-1137 (October-November 2010)

\begin{abstract}
We consider a new type of cooling mechanism for a suspended nanowire acting as a weak link between two superconductive electrodes. By applying a bias voltage over the system, we show that the system can be viewed as a refrigerator for the nanomechanical vibrations, where energy is continuously transferred from the vibrational degrees of freedom to the extended quasiparticle states in the leads through the periodic modulation of the inter-Andreev level separation. The necessary coupling between the electronic and mechanical degrees of freedom responsible for this energy-transfer can be achieved both with an external magnetic or electrical field, and is shown to lead to an effective cooling of the vibrating nanowire. Using realistic parameters for a suspended nanowire in the form of a metallic carbon nanotube we analyze the evolution of the density matrix and demonstrate the possibility of cooling the system down to a stationary vibron population of $\sim 0.1$. Furthermore, it is shown that the stationary occupancy of the vibrational modes of the nanowire can be directly probed using the dc current responsible for carrying away the absorbed energy from the vibrating nanowire. () 2010 American Institute of Physics.
\end{abstract}

[doi:10.1063/1.3515521]

\section{INTRODUCTION}

Nanoelectromechanical systems (NEMS) have over the last two decades been a very active field of both fundamental and applied research. These systems have typical dimensions on the nanoscale and combine electronic and mechanical degrees of freedom for novel applications. These include ultra sensitive mass detection ${ }^{1-3}$ and position sensing ${ }^{4-6}$ using small mechanical resonators with a resolution that has been shown to approach the limit set by the Heisenberg uncertainty principle. Due to the small dimensions implied by NEMS they border on the world of quantum mechanics while still being macroscopic in the sense that they can be fabricated lithographically. Nanoelectromechanical systems thus allow for the controlled fabrication of devices whose physics is ultimately governed by the fundamental limits set by quantum mechanics. Achieving control and understanding of these systems is therefore of great interest as doing so opens up a whole new toolbox for the design of highperformance applications. ${ }^{7,8}$

Typically, NEMS comprise a mechanical resonator in the form of a cantilever or a doubly-clamped beam coupled to an electronic system used for both actuation and detection. Due to the high mechanical vibration frequencies and exceptionally high quality factors recently achieved, these systems allow for very low energy dissipation and extreme sensitivity to external stimuli. To make full use of their potential much research has recently focused on the possibility of effectively cooling the mechanical subsystem in NEMS to its vibrational ground state, which would enable unprecedented mass- and position-sensitivity of the resonators. Recently, O'Connell and colleagues ${ }^{9}$ showed that complete ground state cooling of a mechanical resonator can be achieved at very low temperatures. In Ref. 9 the reported vibron occupancy factor is $<0.07$, which means the resonator is in its ground state with a probability of $93 \%$, well within the quantum regime. Here we note, however, that this effect is attributed to the high mechanical frequency of the resonator (in the $\mathrm{GHz}$ range) and the very low $(25 \mathrm{mK})$ temperature of the dilution fridge. No active cooling of the resonator is needed as the low occupancy of its excited vibrational modes simply follows from the Bose-Einstein distribution function at the equilibrium temperature. To date, the best experimental results for active cooling of a mechanical resonator lead to a vibron occupation factor of 3.8, as recently reported by Rocheleau et al. ${ }^{10}$ In Ref. 10 an external electromagnetic field acts as an energy transducer for side-band cooling of the mechanical resonator (see also Ref. 11, where dynamical back-action is used to achieve similar results).

In this paper we consider a new experimental setup for ground state cooling of a mechanical resonator, a nanomechanical superconducting weak link as shown schematically in Fig. 1. Below we show that this system can be viewed as 
$\mathrm{L}$

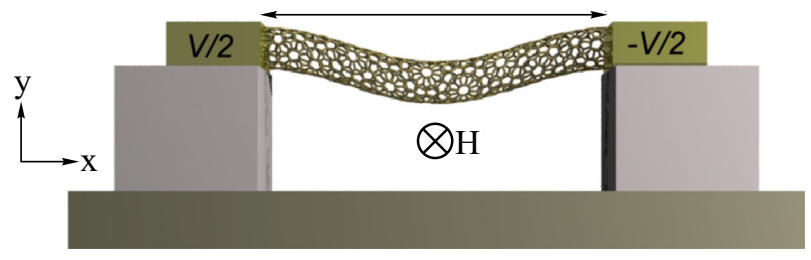

FIG. 1. (Color online) Schematic diagram of the system considered. A suspended carbon nanotube of length $L$ is coupled to two superconducting leads biased at a voltage $V$. Coupling between the Andreev states for the electronic degrees of freedom and the mechanical vibrations of the nanowire is achieved through the external transverse magnetic field $H$ which enables transition between the electronic branches through the emission/absorption of a vibrational quantum (see text).

a refrigerator for the nanomechanical vibrations, where the voltage-driven Andreev states serve as the refrigerant responsible for pumping energy from the nanomechanical vibrations to the thermostat of quasiparticle states in the leads. The possibility of such an energy transfer is based on the periodic, in time, modulation of the inter-Andreev level spacing and the coupling between these current carrying Andreev states and the mechanical degrees of freedom through an applied magnetic field (we note that this coupling could also be achieved through an external electric field as outlined in Appendix A). At the start of each cooling cycle the Andreev level separation initially shrinks, which brings them into thermal contact with the vibronic degrees of freedom, enabling energy exchange between the two systems. During this time, the work done by the bias voltage results in an «over-cooling» of the Andreev level population, which makes the heat transfer from the nanomechanical to the electronic degrees of freedom possible. After a while the interAndreev level separation increases again, eventually reaching its initial value, at which point the Andreev states release their excess energy into the extended quasiparticle states of the leads. The cooling process suggested here thus models a nanomechanical refrigerator where heat is continuously transferred along the chain; nanomechanical vibrations $\rightarrow$ Andreev levels $\rightarrow$ quasiparticle states, resulting in an overall effective cooling of the nanomechanical vibrations.

Unlike many recent theoretical models in which electromechanically induced ground state cooling of mechanical resonators has been reported, ${ }^{12-16}$ the cooling mechanism suggested here relies neither on the abovementioned sideband effect nor on dynamical back-action. Rather, the cooling is an inherent property of the device which makes the suggested mechanism a promising candidate for truly quantum mechanical manipulation of mechanical resonators.

The electromechanical coupling required for our cooling mechanism to work will be assumed to be due to an external magnetic field applied perpendicular to the length of the nanowire. ${ }^{17}$ Displacements of the nanowire will then couple the mechanical and electronic degrees of freedom through the Lorentz force. For a «short» SNS junction (with a coherence length much longer than the nanowire) the ac Josephson dynamics of the device, induced by a weak dc driving voltage, $e V<(\Delta E(\phi))^{2} / \Delta_{0}$, can be expressed in terms of a pair of Andreev states, which are periodically created and destroyed in the junction. Here, $\Delta E(\phi)$ is the distance between

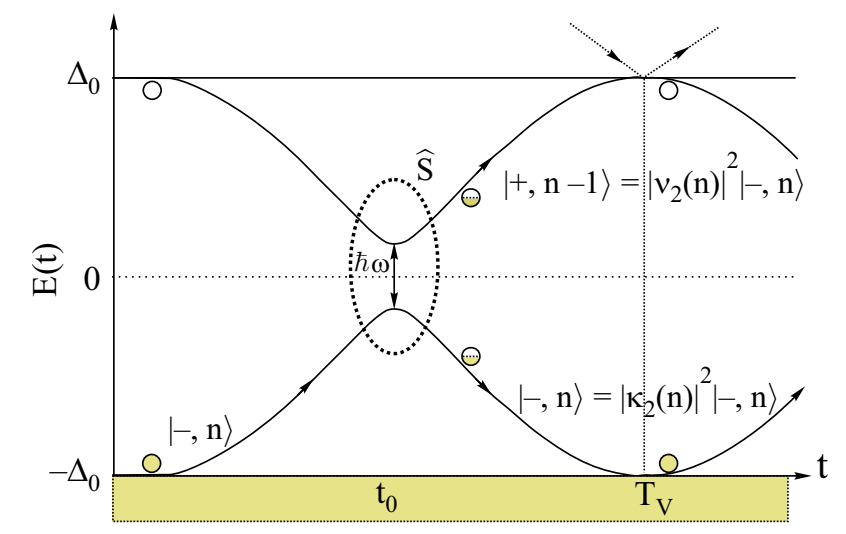

FIG. 2. Time evolution of the Andreev states (full lines) over one period $T_{V}=\pi \hbar / \mathrm{eV}$. The state of the total system, $| \pm, n\rangle$, depends on the population of the two electronic branches, $|+\rangle$, corresponding to the upper and lower branch, respectively, as well as on the quantum state of the oscillating nanowire, $|n\rangle$. Due to the large separation in energies between $\Delta_{0}$ and $\hbar \omega$, transitions between the electronic branches is only possible in the small resonance-window $t_{0}-\delta t \leqslant t \leqslant t_{0}+\delta t$ (modeled through the scattering matrix $\hat{S}$ ) where the electronic state of the system can change through the emission/ absorption of one vibrational quantum. In the above, the probability for the state initially in $|-, n\rangle$ to scatter into the state $|+, n-1\rangle$ after passing through the resonance depends on the state of the oscillator through the coefficient $\left|\nu_{2}(n)\right|^{2}$ (see text). After one period the partially filled Andreev levels join the continuum, a process which is here represented by dashed arrows, and the electronic states are reset (filled and empty circles).

the Andreev levels, $\phi=\phi(t)$ is the voltage-driven phase difference over the junction, and $\Delta_{0}$ is the order parameter in the superconductors. The creation/destruction of the Andreev states takes place at the edges of the superconducting gap, $\Delta E=2 \Delta_{0}$, when $\phi(t)=2 \pi n$ (see Fig. 2). Between creation and destruction the Andreev levels evolve adiabatically within the energy gap of the superconductors, such that the inter-Andreev level spacing first shrinks and is later restored to its initial value $2 \Delta_{0}$ at a time when the Andreev states dissolve in the continuum quasiparticle spectrum (see Fig. 2). The whole cycle then repeats itself again and again.

Being thermally populated at the moment of their nucleation, the Andreev states will initially, as the interlevel spacing decreases, experience an effective cooling given that the thermal relaxation is not fast enough to follow the level displacement. Under such conditions, the Andreev states can accumulate energy from any external system (in particular from the nanomechanical vibrations) if interactions with such a system are established. The absorbed energy is then transferred into the electronic quasiparticle continuum when the Andreev states are dissolved and the process of cooling of the external system is continued through the formation of new thermally populated Andreev states, which in their turn evolve with time and absorb energy. After each full period of evolution the extra energy absorbed in this process is removed from the SNS junction by the quasiparticle excitations.

Besides being a new type of system where efficient cooling of nanomechanical-resonators can be achieved, this type of superconducting weak links makes it possible to couple the nanomechanical system of the device to the macroscopically coherent superconducting states. It presents an exciting possibility of entangling the current carrying Andreev states associated with the SNS junction with the quantum states of 
nanomechanical vibration when the mechanical subsystem is sufficiently cooled. Manipulation of such nanoelectromechanical coherent states can be done both electronically, by means of driving- and gate voltages, or mechanically, by controlling the displacement of a movable part of the device.

\section{MODEL HAMILTONIAN}

To discuss the cooling mechanism quantitatively we use the Hamiltonian,

$$
\begin{aligned}
& \hat{H}(t)=\hat{H}_{\mathrm{el}}+\hat{H}_{\mathrm{mech}}+\hat{H}_{\mathrm{int}}, \\
& \hat{H}_{\mathrm{el}}(t)=\left(\begin{array}{cc}
-\frac{\hbar^{2}}{2 m} \frac{\partial^{2}}{\partial x^{2}}-\mu & \Delta(x) \mathrm{e}^{i \phi(t)} \\
\Delta(x) \mathrm{e}^{-i \phi(t)} & \frac{\hbar^{2}}{2 m} \frac{\partial^{2}}{\partial x^{2}}+\mu
\end{array}\right), \\
& \hat{H}_{\text {mech }}=\hbar \omega \hat{b}^{\dagger} \hat{b} .
\end{aligned}
$$

In $(1), \hat{H}_{\mathrm{el}}(t)$ is the Bogoliubov-de Gennes (BdG) Hamiltonian for the electronic degrees of freedom. Here, $\Delta(x)$ $=\Delta_{0} \Theta(|x|-L / 2)$ is the order parameter in the superconductive leads (here taken to be identical), $\mu$ is the chemical potential, and $\phi(t)$ is the phase difference over the junction, which depends on the bias voltage $V$ according to the Josephson relation $\dot{\phi}=2 \mathrm{eV} / \hbar$. The second term in the Hamiltonian describes the oscillating nanowire which is modeled as a quantum mechanical harmonic oscillator with only the fundamental mode excited; $\hat{b}^{\dagger}[\hat{b}]$ is an operator that creates [annihilates] a quantum of vibration and $\omega$ is the frequency of the fundamental mode. In (1), $\hat{H}_{\text {int }}$ is the interaction Hamiltonian which gives the coupling between the electronic and the mechanical degrees of freedom through the magnetic field.

At the superconductor-nanowire interface two electronic scattering processes are possible, viz. normal reflection and Andreev reflection. These give rise to two localized statesAndreev states-described by the wavefunctions which satisfy $\hat{H}_{\mathrm{el}} \psi_{ \pm}(x, t)=E_{ \pm} \psi_{ \pm}(x, t)$ where $\psi_{ \pm}(x, t)$ are two-state spinors in Nambu space, whose eigenenergies are given by the expression $E_{ \pm}(t)= \pm \Delta_{0} \times\left[1-D \sin ^{2}(\phi(t) / 2)\right]^{1 / 2}$, where $D=1-R$ is the normal junction transparency. ${ }^{18,19}$ These Andreev states are responsible for carrying the Josephson supercurrent through the (normal) nanowire. Under the condition that these states evolve slowly in time $\left(\hbar \dot{\phi} \ll \Delta_{0}\right)$, they describe a two-level system for the electronic degrees of freedom. As the Andreev states carry current through the oscillating nanowire, they will couple the electronic degrees of freedom to the mechanical degrees of freedom through the Lorentz force. It is thus more natural to describe the system in terms of the temporally evolving Andreev states, to which purpose we rewrite the Hamiltonian as

$$
\begin{aligned}
\hat{H}(t)= & \mathcal{E}(\phi(t)) \hat{\sigma}_{z}+\Delta_{0} \sqrt{R} \sin (\phi(t) / 2) \hat{\sigma}_{x}+\hbar \omega \hat{b}^{\dagger} \hat{b} \\
& +\frac{2 e}{\hbar} \frac{\partial \mathcal{E}(\phi(t))}{\partial \phi} L H \hat{y} \hat{\sigma}_{z} .
\end{aligned}
$$

Here, $\mathcal{E}(\phi(t))$ is the energy of the Andreev states for the completely transparent $(R=0)$ junction and $\hat{\sigma}_{i}(i=x, y, z)$ are the Pauli matrices. The last term in (4) describes the electromechanical coupling of the current-carrying Andreev states to the motion of the nanowire through the Lorentz force. Here, $e$ is the electronic charge, $L$ is the length of the wire, $\hat{y}=y_{0}\left(\hat{b}^{\dagger}+\hat{b}\right)$-where $y_{0}$ is the zero-point oscillation amplitude-is the deflection operator in the transverse $y$-direction, $H$ is the magnetic field and $2 e / \hbar(\partial \mathcal{E} / \partial \phi) \hat{\sigma}_{z}$ is the current operator in the nanowire. ${ }^{20}$

From Fig. 2 one can see that the cooling mechanism described is maximally efficient if the strongest coupling of the mechanical subsystem to the Andreev states occur during the time interval when the Andreev levels are closest to the chemical potential, i.e., when the population of the Andreev levels differ most from what it would be in thermodynamic equilibrium. The coupling is resonant during this time if $\hbar \omega=\min (\Delta E(\phi))=2 \Delta_{0} \sqrt{R}$ (where $R=1-D$ is the normalstate reflection probability), which requires the weak link to have a high normal-state transparency $D$ for electrons. ${ }^{21}$ This is because, for the system considered, the energy-scales of the superconductive order parameter and the mechanical vibrations, $\hbar \omega$, are very different. Also, one notes that when $\phi=\pi$, the Andreev states $\psi_{ \pm}(\phi=\pi)$, with energies $E_{ \pm}(\phi$ $=\pi)= \pm \Delta_{0} R^{1 / 2}$, are symmetric/antisymmetric superpositions of states carrying current in opposite directions. Transitions between the Andreev states-induced by the nanowire as it vibrates in a transverse magnetic field - are therefore by far most probable when $\phi\left(t=t_{0}\right)=\pi$.

In the adiabatic regime, i.e., when $e V \leqslant e V_{c}=4 R \Delta_{0}$, it is convenient to switch to the basis set formed by the Andreev states $\psi_{ \pm}(\phi(t)) .{ }^{1)}$ In this basis the Hamiltonian reads,

$$
\begin{aligned}
\hat{H}_{\text {eff }}(t)= & \left\langle\psi_{ \pm}|\hat{H}(t)| \psi_{ \pm}\right\rangle=E(t) \hat{\tau}_{z}+\hbar \omega \hat{b}^{\dagger} \hat{b} \\
& +\Delta_{0} \Phi\left(\hat{b}^{\dagger}+\hat{b}\right) \hat{\tau}_{x} .
\end{aligned}
$$

Here, the Pauli matrices $\hat{\tau}_{i}$ span the space formed by the states $\psi_{ \pm}(\phi)$. In the last term of (5), $\Phi=2 L H \pi y_{0} / \Phi_{0}$ is the dimensionless magnetic flux threading the area swept by the nanowire as it vibrates in the ground state; $\Phi_{0}=h / 2 e$ is the magnetic flux quantum. Below we will consider the resonant situation when $\hbar \omega=2 \Delta_{0} R^{1 / 2}$, which is the optimum condition for the proposed cooling mechanism as outlined above. ${ }^{2)}$

The Hamiltonian (5) describes the evolution of the Andreev states. Coupling between these is achieved through the off-diagonal terms in (5). These are proportional to $\Phi$ and become relevant whenever the energy separation between the electronic branches matches the energy scale set by the oscillating nanowire. At such times, transitions between the Andreev states can occur through the absorption/emission of one vibrational quantum. This is shown in Fig. 2 for the case when only the lowest electronic branch is initially populated.

\section{MAGNETIC FIELD INDUCED ANDREEV STATE SCATTERING}

Assuming the conditions for adiabaticity outlined above are fulfilled, we now evaluate the probability of mechanically induced Andreev level transitions as a function of the coupling strength. Due to the large separation of energy scales, $2 \Delta_{0} \gg \hbar \omega$, it is not possible to evaluate such transitions using the Landau-Zener formalism ${ }^{20,22}$ as this assumes a linear expansion of $E(t)$ around the resonance point. 
Rather, we consider transitions only to occur in the vicinity of $t_{0}=T_{V} / 2=\pi \hbar / 2 \mathrm{eV}$ and evaluate (5) using a parabolic expansion of $E(t)$. From (5), the dimensionless time-dependent Schrödinger equation for the probability amplitudes $c_{ \pm, n}(\tau)$ of finding the state of the system in the upper/lower electronic branch with the oscillator in the state $n$ reads,

$$
\begin{gathered}
i \partial_{\tau} c_{+, n-1}(\tau)=\tau^{2} c_{+, n-1}(\tau)+\Gamma\left(\sqrt{n} c_{-, n}(\tau)\right. \\
\left.+\sqrt{n-1} \mathrm{e}^{2 i \lambda \tau} c_{-, n-2}(\tau)\right) \\
i \partial_{\tau} c_{-, n}(\tau)=-\tau^{2} c_{-, n}(\tau)+\Gamma\left(\sqrt{n} c_{+, n-1}(\tau)\right. \\
\left.+\sqrt{n+1} \mathrm{e}^{-2 i \lambda \tau} c_{+, n+1}(\tau)\right) ; \\
\tau=\left(t-t_{0}\right)\left(\frac{\xi}{\hbar}\right)^{1 / 3}, \quad \Gamma=\frac{\Phi \Delta_{0}}{\hbar \omega}\left(\frac{V_{c}}{V}\right)^{2 / 3}, \\
\xi=\left.\frac{\partial^{2} E(t)}{\partial t^{2}}\right|_{t_{0}}=\frac{D(\hbar \omega)^{3}}{\hbar^{2}}\left(\frac{V}{V_{c}}\right)^{2}, \\
\lambda \tau=\tau\left(\frac{V_{c}}{V}\right)^{2 / 3}+\frac{\pi \hbar \omega}{2 e V} .
\end{gathered}
$$

Equation (6) describes the adiabatic evolution of the electronic Andreev states. As theses states pass through the resonance they couple to the mechanical subsystem, enabling transitions between them through the emission/absorption of a quantum of vibration as indicated by the terms proportional to the dimensionless electron-vibron coupling constant $\Gamma$ in (6). Considering for now the situation when the system initially starts in the lower electronic branch (6a) we analyze the probability that the system, after passing through $t_{0}$, scatters into the upper electronic branch as a function of the coupling strength. As can be seen from (6a), two scattering processes are possible; absorption of a mechanical vibron (proportional to $\sqrt{n}$ ) or emission of a vibron (proportional to $\sqrt{n-1})$. Note however, that these two processes differ by an exponential phase factor associated with the emission process.

Considering that the coupling between the electronic and mechanical degrees of freedom is very weak (see below) we evaluate the probability for the electronic subsystem to scatter into the upper electronic branch perturbatively in the small parameter $\Gamma$. From this analysis we find that scattering is much more likely to occur through the absorption process than through the emission process, whose importance is reduced by the exponential phase factor mentioned above. A numerical analysis shows that the probability of magneticfield induced Andreev state scattering through the emission channel is only $\sim 3 \%$ of the probability of scattering through the absorption channel. For the case when the system is initially in the upper electronic state (6b) the same analysis shows that the emission process (proportional to $\sqrt{n}$ ) is much more likely than the absorption process (proportional to $\sqrt{n+1})$. Thus, we can safely treat the absorption process (when the electronic system is initially in the lower electronic branch) as the most dominant process, i.e., we may apply the rotating wave approximation (RWA) to (6). Note, however, that without thermal damping of the nanowire vibrations caused by the external environment (see below), the rate of emission would ultimately limit the efficiency of our cooling process as it would prohibit complete ground state cooling. The details of this analysis are given in Appendix B, where we show, without using the rotating wave approximation, that the lowest theoretically achievable level of cooling of the nanowire corresponds to a population of the vibrational mode of $\langle n\rangle \simeq 0.03$.

Within the rotating wave approximation we proceed to evaluate the scattering probability as a function of the coupling strength in more detail, only keeping the terms proportional to $\sqrt{n}$ in (3),

$$
\begin{aligned}
& i \partial_{\tau} \mathbf{c}_{n}(\tau)=\left(\tau^{2} \tau_{z}+\Gamma \sqrt{n} \tau_{x}\right) \mathbf{c}_{n}(\tau), \\
& \mathbf{c}_{n}(\tau)=\left[c_{+, n-1}(\tau), c_{-, n}(\tau)\right]^{T}
\end{aligned}
$$

The electronic scattering mechanism considered here is similar to the Landau-Zener formalism in that the probability of scattering depends on the ratio between the coupling strength and the rate of evolution through the interaction region, off-diagonal terms in (7). However, unlike in the Landau-Zener approach, where in the corresponding equation the dependence on $\tau$ is only linear, equation (7) cannot be solved analytically. Instead we have performed numerical simulations in order to find the probability of scattering as a function of the coupling strength. From this analysis we find that unlike the Landau-Zener formula, which gives a unitary transition probability in the strong coupling limit, the parabolic dependence on $\tau$ in (7) dictates that the probability of transition in the limit of strong coupling (or infinitely slow transition through the interaction region) is only 0.5 . Thus, for very low applied bias voltages we should expect a probability of transition between the Andreev states of 0.5 each time the system passes through the resonance.

In the adiabatic regime, $V \lesssim V_{c}$ the coupling for realistic experimental parameters is, however, relatively weak: $\Gamma$ $=\Delta_{0} \Phi /(\hbar \omega) \ll 1$, hence we can analyze Eq. (7) by treating $\Gamma$ as a perturbation to the solution $\left|c_{-, n}(-\delta \tau)\right|^{2}=1$, where the system starts in the lower electronic branch at time $-\delta \tau=$ $-\delta t(\xi / \hbar)^{1 / 3}$. Here, $\delta t \simeq\left(\hbar \omega \Phi / \Delta_{0}\right)^{1 / 2} \hbar /(e V) \ll \pi \hbar /(e V)$ is the characteristic time scale of the electromechanical interactions. Solving (7) under this condition for the probability $\left|c_{+, n-1}(\delta \tau)\right|^{2}$ of the system's being in the upper branch after passing through the resonance, we find $\left|c_{+, n-1}(\delta \tau)\right|^{2} \simeq n \pi \Gamma^{2}$, which is consistent with our numerical solutions. Thus, for weak coupling between the electronic and mechanical degrees of freedom, the probability for a transition between the Andreev states to have occurred after passing through the resonance (with the corresponding absorption of a mechanical vibron) scales linearly with the initial population of the mechanical resonator. Furthermore, as can be seen from the symmetry of (7), this expression also gives the probability of finding the system in the state $|-, n\rangle$ given that it was nucleated in $|+, n-1\rangle$.

\section{GROUND STATE COOLING OF OSCILLATING NANOWIRE}

In order to model the full evolution of the coupled electromechanical system we evaluate the total density matrix $\hat{\rho}$ of the system over one period. Under the assumption that the thermal energy at the ambient temperature, $T$, is small com- 
pared to the initial separation between the electronic branches, $2 \Delta_{0} / k_{B} T>1$, the system will, at the start of the period, be found in the lower electronic branch with a distribution, $P_{n}^{\text {in }}$, of the mechanical excitations,

$$
\hat{\rho}^{\text {in }}=\sum_{i, j= \pm} \sum_{n=0}^{\infty} P_{n}^{\text {in }}|i, n\rangle\left\langle j, n\left|=\sum_{n=0}^{\infty} P_{n}^{\text {in }}\left(\begin{array}{ll}
0 & 0 \\
0 & 1
\end{array}\right)\right| n\right\rangle\langle n| .
$$

During the adiabatic evolution (no coupling between branches) the system interacts with the external heat bath and the rate of change of the density matrix is given by,

$$
\frac{\partial \hat{\rho}(t)}{\partial t}=-\frac{i}{\hbar}\left[\hat{H}_{\text {eff }}(t), \hat{\rho}(t)\right]+\frac{\gamma}{2} \hat{\mathfrak{L}}(\hat{\rho}(t)),
$$

where the collision integral

$$
\begin{aligned}
\hat{\mathfrak{L}}(\hat{\rho})= & -\left(1+n_{B}\right)\left(\hat{b}^{\dagger} \hat{b} \hat{\rho}+\hat{\rho} \hat{b}^{\dagger} \hat{b}-2 \hat{b} \hat{\rho} \hat{b}^{\dagger}\right)-n_{B}\left(\hat{b} \hat{b}^{\dagger} \hat{\rho}\right. \\
& \left.+\hat{\rho} \hat{b} \hat{b}^{\dagger}-2 \hat{b}^{\dagger} \hat{\rho} \hat{b}\right),
\end{aligned}
$$

models the interaction of the mechanical subsystem with the environment. Here, $n_{B}=\left(\exp \left(\hbar \omega / k_{B} T\right)-1\right)^{-1}$ and $\gamma=\omega / Q$ is the thermal damping rate of the vibrational modes with $Q$ the quality factor. Considering the coupling to the environment to be small $\left(Q \sim 10^{523}\right)$, we can solve for the evolution of the density matrix by treating the interaction of the system with the external environment as a perturbation.

The adiabatic evolution of the electronic system ensures that the coupling between the Andreev states is virtually zero. Only in the small region around $t_{0}$ do the two branches interact (through the coupling to the mechanical degrees of freedom), which is conveniently accounted for by describing the evolution of $\hat{\rho}$ through the resonance by the unitary scattering matrix $\hat{S}$,

$$
\begin{aligned}
& \hat{\rho}\left(t_{0}+\delta t\right)=\hat{S} \hat{\rho}\left(t_{0}-\delta t\right) \hat{S}^{\dagger}, \\
& \hat{S}=\left(\begin{array}{cc}
\kappa_{1}(\hat{n}) & i \frac{\nu_{1}(\hat{n})}{\sqrt{\hat{n}+1}} \hat{b} \\
i \hat{b}^{\dagger} \frac{\nu_{2}(\hat{n}+1)}{\sqrt{\hat{n}+1}} & \kappa_{2}(\hat{n})
\end{array}\right), \\
& \left|\kappa_{i}(n)\right|^{2}+\left|\nu_{i}(n)\right|^{2}=1 .
\end{aligned}
$$

Here, $\hat{n}=\hat{b}^{\dagger} \hat{b}$ is the vibron number operator and the subscripts 1,2 refer to the top/bottom Andreev state, respectively. The coefficients $\kappa_{i}(\hat{n})\left[\nu_{i}(\hat{n})\right]$ are respectively the probability amplitude for the system to stay [scatter] out of the initial Andreev state $i$, both of which depend on the state of the oscillator as outlined above. As such, $\left|\nu_{2}(n)\right|^{2}$ is the probability of the system, initially in the state $n$ in the lower electronic branch, to scatter into the upper electronic branch through the absorption of a vibron. It thus corresponds to $\left|c_{+, n-1}(\delta \tau)\right|^{2}$ from (7) with the initial state $|-, n\rangle$. With this we have $\left|\nu_{2}(n)\right|^{2} \simeq \pi n \Gamma^{2}$ and also $\left|\nu_{2}(n)\right|^{2}=\left|\nu_{1}(n-1)\right|^{2}$ which follows from the symmetry of (7).

After one period the partially filled Andreev branches merge with the superconducting continuum. Here, nonadiabatic interactions release the energy of the chargecarrying quasiparticles into the continuum and the initial conditions for the Andreev level population is reset, ${ }^{22}$ i.e.,

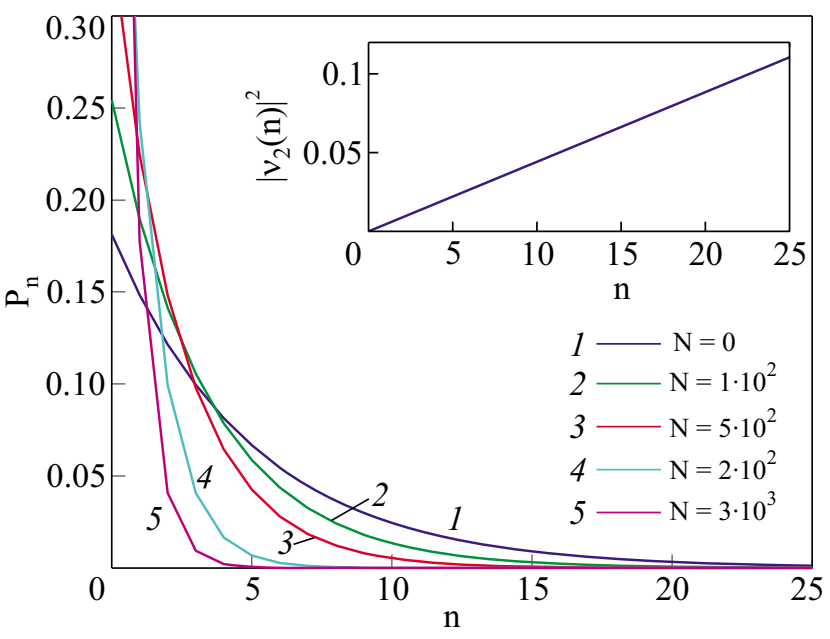

FIG. 3. (Color online). Evolution of the distribution of the mechanical modes, $P_{n}$, as a function of the quantum state $n$ for different number of periods $N\left(T_{V}=\pi \hbar / e V \sim 20 \mathrm{~ns}\right)$. Initially $P_{n}$ is thermally distributed $P_{n}$ $\propto \exp \left(-\hbar \omega n / k_{B} T\right)$ with $k_{B} T=5 \hbar \omega$. Here, $V=10^{-7} \mathrm{~V}, \hbar \omega=10^{-6} \mathrm{eV}, \Delta_{0}$ $=10 \hbar \omega, y_{0}=20 \mathrm{pm}, L=100 \mathrm{~nm}$ and $H=1 \mathrm{~T}$. Inset shows $\left|\nu_{2}(n)\right|^{2}$ as a function of $n$ for the same parameters.

the electronic system returns to the lower branch (see Fig. 2). The mechanical system, of major interest in this paper will not, however, return to the initial distribution after one period. Thus, we may find the density matrix for the mechanical system after one period, $\hat{\rho}_{\text {mech }}^{f}$, by tracing out the electronic degrees of freedom of the total density matrix,

$$
\begin{aligned}
\hat{\rho}^{f}=\sum_{n=0}^{\infty} P_{n}^{f}\left(\begin{array}{ll}
0 & 0 \\
0 & 1
\end{array}\right)|n\rangle\langle n|, \\
\hat{\rho}_{\text {mech }}^{f}=\operatorname{Tr}_{\mathrm{el}}\left(\hat{\rho}^{f}\right)=\operatorname{Tr}_{\mathrm{el}}\left[\hat{S} \hat{\rho}^{\mathrm{in}} \hat{S}^{\dagger}+\frac{\gamma}{2} \frac{T_{V}}{2} \hat{S} \hat{\mathfrak{L}}\left(\hat{\rho}^{\mathrm{in}}\right) \hat{S}^{\dagger}\right. \\
\left.\quad+\frac{\gamma}{2} \frac{T_{V}}{2} \hat{\mathfrak{L}}\left(\hat{S} \hat{\rho}^{\mathrm{in}} \hat{S}^{\dagger}\right)\right] .
\end{aligned}
$$

Equation (12) describes the evolution of the mechanical density matrix over one period. Without coupling to the environment, $\gamma=0$, this corresponds to the decay of the mechanical subsystem, as for each period there is a probability $\left|\nu_{2}(n)\right|^{2}$ that the state $|-, n\rangle$ scatters into the state $|+, n-1\rangle$. Since the opposite process is forbidden if initially only the lower electronic branch is populated, the mechanical subsystem would thus approach the vibrational ground state at a rate which depends on the strength of the coupling. As noted above, complete ground state cooling would, however, not be possible due to the small but finite probability of interAndreev level scattering through the emission channel.

When effects of the environment are included, the mechanical subsystem does not necessarily decay to the ground state since the external damping drives the system towards thermal equilibrium. In Fig. 3 we plot the evolution of the distribution of the vibrational states, $P_{n}$, as a function of the quantum numbers $n$ over many periods for realistic experimental parameters. As can clearly be seen, the above scattering process acts to lower the initially thermally distributed population of the mechanical system, effectively cooling the nanowire down to a final population of the vibronic states of 
$\langle n\rangle=\Sigma_{n} P_{n} n \sim 0.1$. Note, however, that complete ground state cooling is not achieved due to the temperature of the external environment.

To evaluate the efficiency of this cooling mechanism we analyze the stationary solution of (12), i.e., when the mechanical subsystem does not change over one period, perturbatively in the small parameter $\gamma$. From this analysis we find that to zeroth order in $\gamma$ the stationary solution gives $\left|\nu_{2}(n)\right|^{2}$ $P_{n}=0$ which corresponds to the distribution of the mechanical modes decaying to the ground state $\left(\left|\nu_{2}(0)\right|^{2}=0\right)$. The corrections to this solution due to the external heat bath are found using the higher order terms in the perturbation expansion where the lowest order correction to the state $n$ scales as $\gamma^{n}$. We thus find that to linear order in $\gamma$ the correction to the distribution of the mechanical modes in the stationary regime, $P_{n}^{\text {stat }}$, is given by

$$
\begin{aligned}
& P_{n}^{\text {stat }}=P_{n}^{0}+\gamma \frac{\pi \hbar n_{B}}{2 e V} \delta P_{n}+O\left(\gamma^{2}\right), \\
& P_{n}^{0}=P_{0}^{2} \delta_{n, 0}, \\
& \delta P_{n}=(-1)^{n+1} \frac{\left(2-\left|\nu_{2}(1)\right|^{2}\right)}{\left|\nu_{2}(1)\right|^{2}}, \quad n=0,1 .
\end{aligned}
$$

With this, we find that the population of the first excited state in the stationary regime can be expressed as,

$$
P_{1}^{\text {stat }} \simeq \frac{n_{B}}{Q \Phi^{2}} \frac{\hbar \omega}{\Delta_{0}}\left(\frac{V}{V_{c}}\right)^{1 / 3} \sim 0.1
$$

which is in accordance with the results shown in Fig. 3.

In this analysis, the strength of the electromechanical coupling, $\Gamma$, plays two roles. First, it dictates the rate of the cooling process as $\left|\nu_{2}(n)\right|^{2} \propto \Gamma^{2}$. Secondly, stronger coupling ensures a lower final distribution of the mechanical modes; in effect, the ratio $\gamma / \Gamma^{2}$ dictates how many terms in the perturbation expansion (13) need to be considered in order to accurately describe the stationary distribution of the mechanical modes. Thus, for the parameters considered, we find that we need to include higher order corrections in $\gamma$ in the expansion (13) to fully describe the stationary distribution. This is confirmed in Fig. 3 which shows a finite probability of not only the ground state and first excited state being populated in the stationary regime. Nevertheless, the expectation value of the population of the mechanical subsystem in Fig. 3 is $\langle n\rangle \simeq 0.1$, which is well in the quantum regime.

\section{DC CURRENT MEASUREMENT AS A PROBE FOR THE STATIONARY VIBRONIC POPULATION}

To probe the stationary distribution of the mechanical subsystem we suggest studying the dc current through the weak link since, as shown below, this directly measures the vibron population. By changing the quality factor of the system, for example by varying the external pressure, we find that the stationary vibron population can be directly measured since it scales linearly with the dc current. To show this we evaluate the dc current at the resonance induced by the inter-Andreev level scattering over one period when the mechanical subsystem has been driven into the stationary regime. This current forms as the two Andreev levels carry

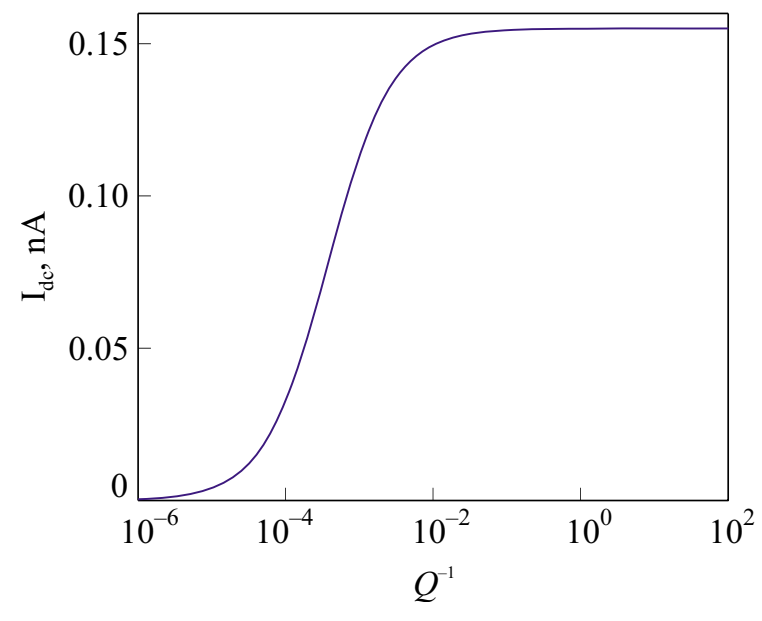

FIG. 4. DC current as a function of $Q^{-1}$ inverse quality factor when the mechanical subsystem has been driven into the stationary regime. As can be seen, in the limit of very high quality factor, corresponding to complete ground state cooling of the oscillating nanowire, the dc current goes zero as no inter-Andreev level scattering is possible in this regime $\left(\left|\nu_{2}(0)\right|^{2}=0\right)$. In the opposite limit, $Q \lesssim 10$, the dc current approaches a constant value which depends on the external temperature, $I_{d c}^{\text {therm }} \propto n_{B}$. Here, the system parameters are the same as in Fig. 3.

current in opposite directions. As such, any scattering event which populates the upper Andreev level will result in a net charge transfer through the system. Evaluating the total current transfer over a full period we have,

$$
\begin{aligned}
I_{d c}= & \frac{2 e}{\hbar} \frac{2}{T_{V}}\left[\int_{0}^{T_{V} / 2} \frac{\partial E_{-}(\phi)}{\partial \phi} d t+(1-P) \int_{T_{V} / 2}^{T_{V}} \frac{\partial E_{-}(\phi)}{\partial \phi} d t\right. \\
& \left.+P \int_{T_{V} / 2}^{T_{V}} \frac{\partial E_{+}(\phi)}{\partial \phi} d t\right],
\end{aligned}
$$

where, $P$ is the total probability for the system to be scattered from the lower to the upper Andreev branch during one period of evolution. Since $n$ vibration quanta are excited with probability $P_{n}^{\text {stat }}$, and since in this case the scattering probability is $\left|\nu_{2}(n)\right|^{2}$, the total scattering probability $P$ is readily obtained, and the expression for $I_{d c}$ simplifies to (note that the time integration is trivial since $\partial / \partial \phi=(\partial t / \partial \phi) \partial / \partial t$ $\left.=\left(T_{v} / 2 \pi\right) \partial / \partial t\right)$

$$
\begin{aligned}
I_{d c} & =\frac{4 e \Delta_{0}}{\pi \hbar}\left(1-\frac{\hbar \omega}{2 \Delta_{0}}\right) \sum_{n=0}^{\infty}\left|\nu_{2}(n)\right|^{2} P_{n}^{\text {stat }} \\
& =e\langle n\rangle \Gamma^{2} \frac{e V_{c}}{\hbar}\left(\frac{1-\sqrt{R}}{R}\right),
\end{aligned}
$$

which is plotted in Fig. 4 for the same experimental parameters as in Fig. 3.

Equation (16) shows that the dc current over the junction scales linearly with the average vibron population in the stationary regime. Here, we note that the correct expression for the current should also take into account corrections from the environment as discussed in Appendix C. Numerical simulations show, however, that the inclusion of these terms does not change the calculated dc current as compared to that shown in Fig. 4.

From Fig. 4 we note that in the limit of small quality factor, i.e., high damping, the dc current saturates to a con- 
stant values. This can be understood by the fact that in this limit the stationary distribution of the vibrational modes is simply given by the thermal distribution $P_{n}^{\text {stat }}$ $\propto \exp \left(-n \hbar \omega / k_{B} T\right)$, i.e., the interactions with the environment are so strong that they always drive the mechanical system into thermal equilibrium, no matter how the inter-Andreev level scattering changes this distribution. As such, $I_{d c} \propto n_{B}$ in this regime. In the opposite regime, $Q \rightarrow \infty$, the dc current goes to zero (within the rotating wave approximation) as expected, since this regime corresponds to complete ground state cooling of the mechanical subsystem. If this can be achieved, the probability of Andreev level scattering also goes to zero, $\left|\nu_{2}(0)\right|^{2}=0$; hence the electronic subsystem stays in the lower Andreev branch throughout and $I_{\mathrm{dc}}=0$.

Based on the dependence of $I_{d c}$ on $1 / Q$ in Fig. 4, we suggest a simple probe by which one could measure the average vibron population once the mechanical subsystem has been driven into the stationary regime. We propose to change the quality factor, for example, by increasing the external pressure of the gas in which the nanowire vibrates, and measure the dc current in the limit of large damping. As $I_{d c}$ scales with the thermal equilibrium phonon population, $n_{B}$, in this regime a further measurement in the low damping regime should thus give a direct measurement of the average vibron population,

$$
\langle n\rangle=\frac{I_{d c}}{I_{d c}^{\text {therm }}} n_{B},
$$

where $I_{d c}^{\text {therm }} \propto n_{B}$ is the dc current in the limit of high damping.

\section{CONCLUSIONS}

To conclude, we have shown that quantum mechanical cooling of a nanomechanical resonator acting as a weak link between two superconductors is possible. In particular, we have considered the example of a suspended carbon nanotube where the unique combination of high resonance frequencies and mechanical quality factors together with high electronic transparency lead to sufficiently strong coupling for efficient cooling to be possible. Using realistic experimental parameters, we have shown that for a short suspended nanotube, stationary distribution of the population of the vibrational modes can be as low as $\langle n\rangle=0.1$ which is truly in the quantum mechanical regime. Furthermore, the suggested mechanism does not rely on any external electromagnetic fields to stimulate the cooling process. Rather, the proposed system should act as a self-cooling device, given the right experimental parameters, as the over-cooled Andreev states can readily absorb energy from the mechanical subsystem provided that sufficient coupling between the two can be achieved. The corresponding energy uptake of the electronic subsystem is later released into the quasiparticle continuum, leading to an effective cooling of the nanomechanical resonator.

Furthermore, it has been shown that by measuring the dc current as a function of the quality factor, the stationary distribution of the population of the vibrational modes can be directly probed. Thus, the suggested system not only has the capacity of self-cooling, but it also presents a direct, internal probe by which to measure the efficiency of the cooling mechanism.

The authors would like to thank Yury Tarakanov for help with graphical illustrations. This work was supported in part by the Swedish VR and SSF, by the EC project QNEMS (FP7-ICT-233952) and by the Korean WCU program funded by MEST through KOSEF (R31-2008-000-10057-0).

\section{APPENDIX A: ELECTRICAL FIELD INDUCED COOLING OF SUSPENDED NANOWIRE}

As mentioned above the required coupling for effective cooling of our suspended vibrating nanowire could also be achieved using a transverse electric field. Under such situations, the non-superconducting suspended wire supplies a coupling of the Josephson current flow to the nanomechanical bending vibrations through the displacement of the wire with the electromechanical coupling depending on the electronic backscattering induced by the electrical field. With this setup, the interaction term in the Hamiltonian (1) reads,

$$
\hat{H}_{\text {int }}=e \mathcal{E} y_{0}\left(\hat{b}^{\dagger}+\hat{b}\right) \hat{\sigma}_{z}
$$

where $\mathcal{E}$ is the transverse electric field. Proceeding as above, we project out the Andreev states $\psi_{ \pm}$, which gives the effective Hamiltonian,

$$
\begin{aligned}
\hat{H}_{\text {eff }}(t)= & \left\langle\psi_{ \pm}|\hat{H}(t)| \psi_{ \pm}\right\rangle=\Delta E(t) \hat{\tau}_{z}+\hbar \omega \hat{b}^{\dagger} \hat{b}+\Xi\left(\hat{b}^{\dagger}+\hat{b}\right) \\
& \times\left(\hat{\tau}_{+} V_{+-}+\text {h.c. }\right), \\
\Delta E(t)= & E(t)+\lambda\left(\hat{b}^{\dagger}+\hat{b}\right) V_{++}, \quad \Xi=e \mathcal{E} y_{0} .
\end{aligned}
$$

In the above, $V_{i, j}=\left\langle\psi_{i}\left|\hat{\sigma}_{z}\right| \psi_{j}\right\rangle, \hat{\tau}_{+}=\left(\hat{\tau}_{x}+i \hat{\tau}_{y}\right) / 2$ and $\left|V_{+-}\right|$ $\propto R .^{22}$ To evaluate this further we perform the unitary transformation $\hat{U} \hat{H}_{\mathrm{eff}}(t) \hat{U}^{\dagger}$,

$$
\hat{U}=\exp \left[\alpha\left(\hat{b}^{\dagger}-\hat{b}\right) \hat{\tau}_{z}\right], \quad \alpha=\Xi V_{++} / \hbar \omega \ll 1,
$$

after which the Hamiltonian reads,

$$
\begin{aligned}
\hat{\mathcal{H}}_{\mathrm{eff}}(t)= & E(t) \hat{\tau}_{z}+\hbar \omega \hat{b}^{\dagger} \hat{b}+\Xi\left(\hat{b}^{\dagger}+\hat{b}\right)\left(V_{+-} \hat{\tau}_{+}+h . c\right) \\
& +O\left(\alpha^{2}\right) .
\end{aligned}
$$

From here on, the analysis follows the previous one with the coupling constant now being, $\Gamma=\left(\Xi\left|V_{+-}\right| / \hbar \omega\right)\left(V_{c} / V\right)^{2 / 3}$. For realistic experimental parameters, our analysis shows that the coupling constant is again much smaller than unity. Hence we can develop the same perturbative analysis for the evolution of the density matrix. Using the same physical parameters as above for the dimensions of the nanowire, the external temperature, and the superconductive gap with $\mathcal{E}$ $=4 \cdot 10^{4} \mathrm{~V} \cdot \mathrm{m}^{-1}$, we find a stationary occupancy of the vibrational modes of the oscillating nanowire of $\langle n\rangle \sim 0.25$. Thus, this analysis shows that an alternative approach to that followed in the main part of this paper would be to use an electric field to provide the electromechanical coupling. Both modes of operation give an efficient cooling of the vibrating nanowire. However, our analysis shows that the best mode of operation for ground state cooling should be to use a magnetic field. This mode also has the added benefit that it makes it possible to tune the superconductive order parameter with the magnetic field to achieve resonant conditions. 


\section{APPENDIX B: EMISSION LIMITED COOLING OF THE NANOWIRE VIBRATIONS}

The analysis presented in the main part of this paper, performed within the rotating wave approximation (RWA), showed that the suggested cooling mechanism has the capacity of effectively reducing the excitations of the nanowire vibrational modes well into the quantum regime. Within the RWA, complete ground state cooling is, in theory, achievable as a large enough quality factor limits the influence of the environment (heating) on the mechanical sub-system. Thus, in the limit $Q \rightarrow \infty$, thermal heating is negligible and the inter-Andreev level scattering mechanism reduces the vibronic population to the ground state with the corresponding decay of $I_{d c}$.

In the rotating wave approximation we ignore the possibility of electronic scattering from the lower to the upper branch through the emission channel. This process can occur if the time spent on resonance, $\delta t$, is short, in which case the uncertainty principle dictates that scattering can occur not only through the absorption but also through the non-energy conserving emission channel. By considering also this channel for inter-Andreev level transition, complete ground state cooling is not achievable (even in the limit $Q \rightarrow \infty$ ) as the emission process will continuously heat the oscillating nanowire. To analyze this we consider (3) in more detail.

Without the benefit of the RWA approximation we need to consider all four off-diagonal terms in (3) in order to fully describe the dynamics of the scattering mechanism. From the form of (6) one can see that, e.g., the term proportional to $\sqrt{n-1}$ corresponds to scattering from the lower electronic branch in the state $n-2$ to the upper electronic branch in the state $n-1$ with emission of a vibron. Thus, this rate of scattering depends on the probability of the occupancy of the harmonic oscillator state $n-2$ and is proportional to $n-1$ as compared to the absorption channel which, as shown above, depends on the probability of the population of the state $n$ and scales linearly with $n$. To take these effects into account we rewrite the unitary scattering matrix (11),

$$
\begin{gathered}
\hat{S}_{2}=\left(\begin{array}{cc}
\kappa_{1}^{\prime}(\hat{n}) & i \frac{\nu_{1}(\hat{n})}{\sqrt{\hat{n}+1}} \hat{b}+i \frac{\chi_{1}(\hat{n})}{\sqrt{\hat{n}}} \hat{b}^{\dagger} \\
i \hat{b}^{\dagger} \frac{\nu_{2}(\hat{n}+1)}{\sqrt{\hat{n}+1}}+i \hat{b} \frac{\chi_{2}(\hat{n}-1)}{\sqrt{\hat{n}}} & \kappa_{2}^{\prime}(\hat{n})
\end{array}\right), \\
\left|\kappa_{i}^{\prime}(n)\right|^{2}=\left|\kappa_{i}(n)\right|^{2}-\left|\chi_{i}(n)\right|^{2}, \\
\left|\kappa_{i}^{\prime}(n)\right|^{2}+\left|\nu_{i}(n)\right|^{2}+\left|\chi_{i}(n)\right|^{2}=1 .
\end{gathered}
$$

In (B1) $\left|\kappa_{2}^{\prime}(n)\right|^{2}$ and $\left|\nu_{2}(n)\right|^{2}$ have the same meaning as before, i.e., they are respectively the probability of staying in or scattering (through the absorption channel) from the lower electronic branch with the oscillator in state $n$. Similarly $\left|\chi_{2}(n)\right|^{2}$ gives the probability that the system scatters from the lower to the upper electronic branch through the emission channel (the subscripts $i=1$ correspond to scattering from the upper to the lower electronic branch). By construction, these rates are,

$$
\begin{aligned}
& \left|\nu_{2}(n)\right|^{2}=\left|\nu_{1}(n-1)\right|^{2}, \\
& \left|\chi_{1}(n)\right|^{2}=\left|\chi_{2}(n-1)\right|^{2},
\end{aligned}
$$

$$
\frac{\left|\chi_{2}(n-2)\right|^{2}}{\left|\nu_{2}(n)\right|^{2}} \frac{n}{n-1} \simeq 0.03,
$$

where $\left|\nu_{2}(n)\right|^{2}=n \pi \Gamma^{2}$ and the coefficient of $n /(n-1)$ in the last line was found from numerical analysis as discussed above.

To analyze the limiting value of the stationary distribution of the vibrational modes in the non-RWA formalism we consider the evolution of the mechanical subsystem with $Q$ $\rightarrow \infty$. Proceeding as above, we evaluate (12) with $\hat{S}=\hat{S}_{2}$ and $\gamma=0$. From this we find that the distribution of the mechanical modes after one period is

$$
P_{n}^{f}=P_{n+1}^{\mathrm{in}}\left|\nu_{2}(n+1)\right|^{2}+P_{n}^{\text {in }}\left|\kappa_{2}^{\prime}(n)\right|^{2}+P_{n-1}^{\text {in }}\left|\chi_{2}(n-1)\right|^{2} .
$$

Equation (B2) dictates that complete ground state cooling of the mechanical sub-system is not achievable as the term proportional to $\left|\chi_{2}(n-1)\right|^{2}$ acts to shift the population to higher vibrational modes and will thus compete with the term proportional to $\mid \nu_{2}(n+1)$ which acts to lower these. Considering, however, that the rate of absorption is much greater than the rate of emission, we find that the stationary solution of (B2) corresponds to a population of the vibrational modes corresponding to $\langle n\rangle=0.03$ which is in accordance with the perturbative solution,

$$
\langle n\rangle \simeq \frac{\left|\chi_{2}(0)\right|^{2}}{\left|\nu_{2}(1)\right|^{2}}=0.03 \text {. }
$$

Thus, we find that by including not only the absorption but also the emission channel for inter-Andreev scattering, the best theoretically achievable level of cooling is given by the ratio of these two rates. The corresponding current with this population of the vibrational modes would be $I_{d c}=1.1 \mathrm{pA}$.

\section{APPENDIX C: ENVIRONMENTAL CORRECTION TO THE DC CURRENT}

As discussed in the main text, the dc current through the nanowire scales with the average vibron population, $\langle n\rangle$. In (16) this is shown for the case when $P=\Sigma_{n}\left|\nu_{2}(n)\right|^{2} P_{n}^{\text {stat }}$. We note here that this expression is not completely valid due to the influence of the external environment. To understand why this is the case we have to consider that the stationary distribution $P_{n}^{\text {stat }}$ gives the vibronic population which does not change over one full period. Over this interval of time, the mechanical subsystem changes in two ways: by temporal evolution and through magnetic-field induced scattering. The value of $P_{n}^{\text {stat }}$ depends on both of these processes. This means that, in order to accurately evaluate the total probability of scattering of the mechanical sub-system, we first have to consider the effects of the environment on the evolution of $P_{n}^{\text {stat }}$ between $0 \leqslant t \leqslant T_{V} / 2$ and calculate the total probability of scattering from this quantity. Performing this analysis we find that the accurate probability of scattering reads,

$$
P=\pi \Gamma^{2}\left[\langle n\rangle-\frac{T_{V}}{2 Q}\left(\langle n\rangle-n_{B}\right)\right],
$$

which is again linearly dependent on $\langle n\rangle$ but also includes a correction term from the environment. 
A numerical analysis of the dc current as a function of $Q$ has been performed using both (16) and (C1), with little or no discrepancy between the two results. To understand why the corrections in (26) do not change $I_{d c}$ we analyze separately the limit of low/high damping which both give $P$ $=\pi \Gamma^{2}\langle n\rangle$. In the limit of high quality factor, the corrections to $P_{n}^{\text {stat }}$ from the thermal interactions are very small $\propto 1 / Q$, hence they do not influence the stationary mechanical distribution over the short time-span $T_{V} / 2$ to any large extent. On the other hand, in the limit of low quality factor the stationary distribution of the mechanical modes is given by the thermal distribution: hence, the correction to the total scattering probability in $(\mathrm{C} 1)$ is identically zero. For intermediate values of $Q$ these competing processes cancel out and the current over the junction depends only on the average vibron population in the stationary regime as given by equation (16).

\footnotetext{
${ }^{a)}$ Email: gustav.sonne@physics.gu.se

${ }^{1)}$ In this regime, the voltage-induced Landau-Zener transition at $\phi=\pi$ between the adiabatic Andreev levels is suppressed, ensuring that without electromechanical coupling the level population remains constant in time.

${ }^{2)}$ This condition can be achieved by controlling the magnitude of the order parameter $\Delta_{0}$ through the magnetic field for junctions with small reflection coefficients.
}

${ }^{1}$ B. Lassagne, D. García-Sánchez, A. Aguasca, and A. Bachtold, Nano Lett. 8, 3735 (2008).

${ }^{2}$ K. Jensen, K. Kim, and A. Zettl, Nat. Nanotechnol. 3, 533 (2008).

${ }^{3}$ A. K. Naik, M. S. Hanary, W. K. Hiebert, X. L. Feng, and M. L. Roukes,
Nat. Nanotechnol. 4, 445 (2009).

${ }^{4}$ M. D. LaHaye, O. Buu, B. Camarota, and K. C. Schwab, Science 304, 74 (2004).

${ }^{5}$ S. Etaki, M. Poot, I. Mahboob, K. Onomitsu, H. Yamaguchi, and H. S. J. van der Zant, Nat. Phys. 4, 785 (2008).

${ }^{6}$ J. B. Hertzberg, T. Rocheleau, T. Ndukum, M. Savva, A. A. Clerk, and K. C. Schwab, Nat. Phys. 6, 213 (2010).

${ }^{7}$ K. C. Schwab and M. L. Roukes, Phys. Today 58, 36 (2005).

${ }^{8}$ M. Blencowe, Phys. Rep. 395, 159 (2004).

${ }^{9}$ A. D. O’Connell, M. Hofheinz, M. Ansmann, R. C. Bialczak, M. Lenander, E. Lucero, M. Neeley, D. Sank, H. Wang, M. Weides, J. Wenner, J. M. Martinis, and A. N. Cleland, Nature 464, 697 (2010).

${ }^{10}$ T. Rocheleau, T. Ndukum, C. Macklin, J. B. Hertzberg, A. A. Clerk, and K. C. Schwab, Nature 463, 72 (2010).

${ }^{11}$ A. Naik, O. Buu, M. D. LaHaye, A. D. Armour, A. A. Clerk, M. P. Blencowe, and K. C. Schwab, Nature 443, 193 (2006).

${ }^{12}$ I. Martin, A. Shnirman, L. Tian, and P. Zoller, Phys. Rev. B 69, 125339 (2004).

${ }^{13}$ S. Zippilli, G. Morigi, and A. Bachtold, Phys. Rev. Lett. 102, 096804 (2009).

${ }^{14}$ I. Wilson-Rae, P. Zoller, and A. Imamoḡlu, Phys. Rev. Lett. 92, 075507 (2004).

${ }^{15}$ S. H. Ouyang, J. Q. You, and F. Nori, Phys. Rev. B 79, 075304 (2009).

${ }^{16}$ S. Zippilli, A. Bachtold, and G. Morigi, Phys. Rev. B 81, 205408 (2010).

${ }^{17}$ G. Sonne, M. E. Peña-Aza, L. Y. Gorelik, R. I. Shekhter, and M. Jonson, Phys. Rev. Lett. 104, 226802 (2010).

${ }^{18}$ P. F. Bagwell, Phys. Rev. B 46, 12573 (1992).

${ }^{19}$ C. W. J. Beenakker, Phys. Rev. Lett. 67, 3836 (1991).

${ }^{20}$ L. Y. Gorelik, V. S. Shumeiko, R. I. Shekhter, G. Wendin, and M. Jonson, Phys. Rev. Lett. 75, 1162 (1995).

${ }^{21}$ J. Kong, E. Yenilmez, T. W. Tombler, W. Kim, H. Dai, R. B. Laughlin, L. Liu, S. Jayanthi, and S. Y. Wu, Phys. Rev. Lett. 87, 106801 (2001).

${ }^{22}$ L. Y. Gorelik, N. I. Lundin, V. S. Shumeiko, R. I. Shekhter, and M. Jonson, Phys. Rev. Lett. 81, 2538 (1998).

${ }^{23}$ A. K. Hüttel, G. A. Steele, B. Witkamp, M. Poot, L. P. Kouwenhoven, and H. S. J. van der Zant, Nano Lett. 9, 2547 (2009).

This article was published in English in the original Russian journal. Reproduced here with stylistic changes by AIP. 